\title{
SUPERMASSIVE DISKS*
}

\author{
R. V. WAGONER** and E. E. SALPETER
}

Cornell University, Ithaca, N.Y., U.S.A.

Abstract. The ultimate source of energy in compact objects is investigated. The model is a highly flattened body of mass $\gtrsim 10^{4} M_{\odot}$, supported by centrifugal force in two directions and by gas and radiation pressure in the third. The properties of this model are compared with those nearly spherical supermassive stars introduced by Hoyle and Fowler.

We would like to discuss some work, done partly in collaboration with James Bardeen (Bardeen and Wagoner, 1969; Wagoner, 1969; Salpeter and Wagoner, 1971), which has been motivated in part by a desire to understand the nature of the ultimate source of the energy produced by the compact objects we have discussed. The type of source

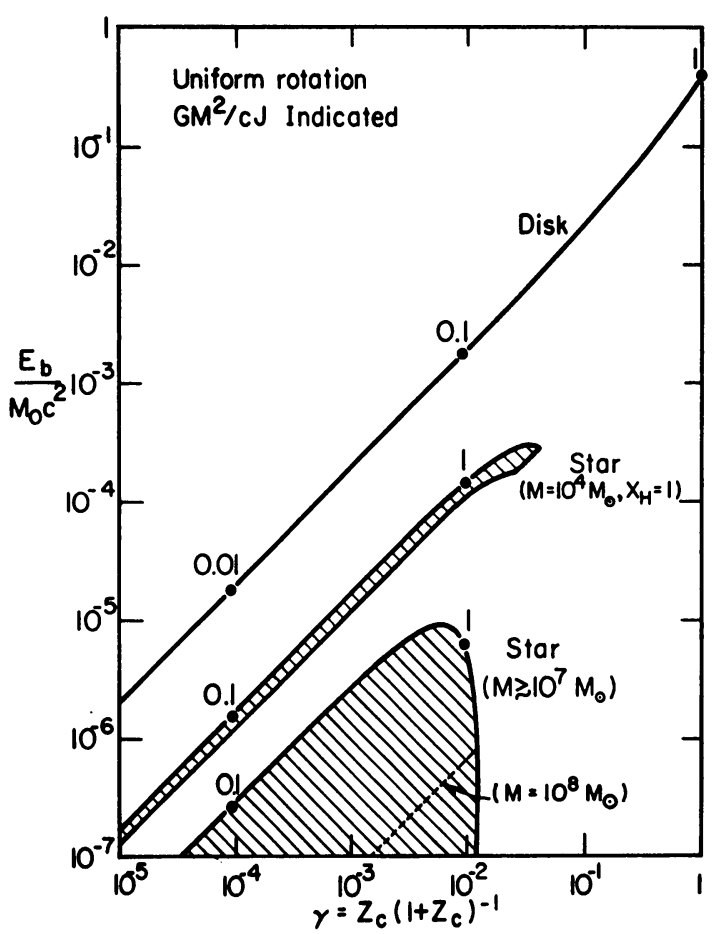

Fig. 1. A comparison of the fractional binding energies of supermassive stars and thin (low entropy) disks as a function of central redshift $z_{c}$.

we shall consider is a highly flattened body of mass $M \gtrsim 10^{4} M_{\odot}$, supported by centrifugal force in two directions and by gas and radiation pressure in the third. We

* Supported in part by the National Science Foundation (GP-9621).

** Alfred P. Sloan Foundation Research Fellow. 
shall compare the properties of such supermassive disks with those of the nearly spherical supermassive stars introduced by Hoyle and Fowler (1963a, b) some seven years ago.

The assumption of uniform rotation is made for mathematical simplicity, but it must be stressed that there are reasons for believing that differential rotation must occur, so that our results probably represent only a qualitative indication of the properties of real disks. However, this is sufficient for our purposes.

The major point of this talk is illustrated on Figure 1, which indicates the domains of quasi-static evolution of uniformly rotating supermassive stars and disks in the fractional binding energy, relativity parameter plane. Here $z_{c}$ is the redshift of the center of the body, and the points indicate various values of the dimensionless rotation parameter $G M^{2} / c J$, which, by comparison, has the value $\sim 10^{-3}$ in typical spiral galaxies.

A supermassive star with $G M^{2} / c J \lesssim 1$ evolves by losing photons until it begins to shed mass at the upper boundary of the shaded region, whereas if $G M^{2} / c J \gtrsim 1$, the star eventually becomes unstable to gravitational collapse at the lower boundary of the region (not shown for $M \gtrsim 10^{7} M_{\odot}$ ). For the mass range of most interest to us, $M \gtrsim 10^{7} M_{\odot}$, the star can reach a binding energy of $E_{b} \approx 10^{-5} M_{0} \mathrm{c}^{2}$ and a central redshift $z_{c} \approx 10^{-2}$. As Fowler (1966) has noted, however, the introduction of differential rotation allows these limits to be increased somewhat.

Let us compare some properties of a supermassive star (with $M \gtrsim 10^{7} M_{\odot}$ ) at the onset of shedding with those of a disk of the same rest mass and angular momentum. We find that the relativity parameter increases by $\sim 10^{2}$, the binding energy increases by $\sim 10^{4}$, the radius decreases by $\sim 10^{2}$, and the central pressure increases by $\sim 10^{8}$ in going from the star to the disk. The fractional binding energy $\rightarrow 0.37$ and $G M^{2} / c J \rightarrow 1$ as the central redshift of the disk approaches infinity. The basic reason for these differences in properties is the fact that the stars are supported by radiation pressure in nearly neutral equilibrium, while the disks are supported by rotation. Although the binding energy of the disks does not reach a maximum at finite $z_{c}$, it is not known whether they are in fact stable against overall gravitational collapse, however. Of course, the well-known smaller scale Newtonian instabilities can be present as well. Nevertheless, it is seen that large rotational energies can at least in principle be made available for conversion into high energy particles and their accompanying radiation through pulsar-like mechanisms (Cavaliere et al., 1969; Morrison, 1969; Cavaliere et al., 1970), for instance.

Note that the disk evolves radially by changes in $J / M^{2}$, whereas the loss of photons only leads to increased flattening with slightly increased binding. In Figure 2 is shown the relation between rotation period and angular momentum $J$ for disks of fixed mass, and the corresponding domains for stars. Unlike the stars, the disks rotate faster as they lose angular momentum. The period of a disk can also be much shorter than that of a star of the same mass.

The properties discussed up to now have been independent of $\beta$, the ratio of gas pressure to total pressure in the disk, as long as it is thin. In Table I are included the 


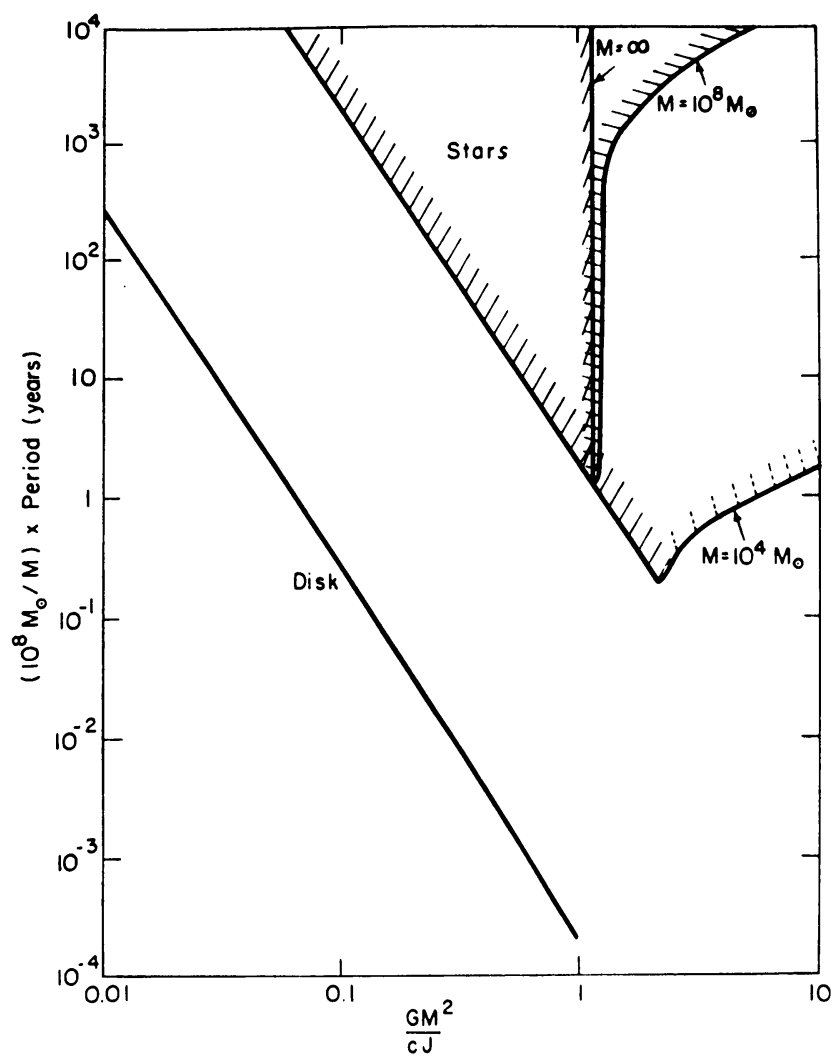

Fig. 2. The relation between the period of rotation, the angular momentum $J$, and the mass $M$ of supermassive stars and disks.

TABLE I

Properties of supermassive objects

$\left(10^{4} \lesssim M / M_{\odot} \equiv \mu \lesssim 10^{12} ; \Omega, S=\right.$ const $\left.; X_{H}=1 ; f_{n}(0)=1\right)$

\begin{tabular}{llll}
\hline Property & Star & Disk & $f_{n}(1)$ \\
\hline$\gamma=Z_{c} /\left(1+Z_{c}\right)$ & $\lesssim 0.012+2.86 \mu^{-1 / 2}$ & $\leqslant 1$ & \\
$2 G M / R c^{2}$ & $\cong 4 / 9 \gamma^{\mathrm{a}}$ & $=8 \gamma f_{1}(\gamma) / 3 \pi$ & 0.78 \\
$G M^{2} / c J$ & $\gtrsim 10.5 \gamma^{1 / 2}$ & $=10 \gamma^{1 / 2} f_{2}(\gamma) / 3 \pi$ & 0.94 \\
$E_{b} / M_{0} c^{2}$ & $\lesssim\left(1.43 \mu^{-1 / 2}+3.0 \times 10^{-3}\right)^{2}$ & $=\gamma f_{3}(\gamma) / 5$ & 1.85 \\
Period $(\mathrm{yr})(=2 \pi / \Omega)$ & $\gtrsim 1.6 \times 10^{-11} \mu \gamma^{-3 / 2}$ & $=2.3 \times 10^{-12} \mu \gamma^{-3 / 2} f_{4}(\gamma)$ & 0.85 \\
$\left(L / L_{\odot}\right) /\left(M / M_{\odot}\right)$ & $=3.2 \times 10^{4} \mathrm{a}$ & $=3.2 \times 10^{4 \mathrm{a}}$ & \\
$\left(T_{9}\right) c$ & $=8.83 \times 10^{3} \mu^{-1 / 2} \gamma^{\mathrm{a}}$ & $=7.12 \times 10^{3} \mu^{-1 / 2} \gamma f_{5}(\gamma)$ & 0.82 \\
$T_{e}(K)$ & $\left.=2.68 \times 10^{5}\left(T_{9}\right)\right)_{c}^{1 / 2 \mathrm{a}}$ & $=5.42 \times 10^{5}\left(T_{9}\right) c^{1 / 2}\left(\sigma / \sigma_{c}\right)^{1 / 4 \mathrm{a}}$ & \\
Entropy $\left(\mathrm{erg} K^{-1} g^{-1}\right)$ & $S_{8}=7.76 \times 10^{7} \mu^{1 / 2 \mathrm{a}}$ & $6.7 \times 10^{8}<S_{d}<S_{8}^{\mathrm{a}}$ & \\
$W / R$ & $\cong 0.7-1.0$ & $=0.20\left(S_{d} / S_{s}\right) f_{6}(\gamma)$ & 0.96 \\
$\beta=P_{g} / P \ll 1$ & $=8.56 \mu^{-1 / 2 \mathrm{a}}$ & $=8.56\left(S_{s} / S_{d}\right) \mu^{-1 / 2 \mathrm{a}}$ & \\
$Q\left(\mathrm{~g} \mathrm{~cm}{ }^{-3}\right)$ & $=1.30 \times 10^{5} \mu^{-1 / 2} T_{9}{ }^{3 \mathrm{a}}$ & $=1.30 \times 10^{5}\left(S_{s} / S_{d}\right) \mu^{-1 / 2} T_{9}{ }^{3 \mathrm{a}}$ & \\
\hline
\end{tabular}

a Relations also valid for differential rotation. 
relations of some of these properties to the mass parameter $\mu$ and the relativity parameter $\gamma$. In order to investigate other properties we now assume that $\beta \ll 1$ within the disks, as is required for the supermassive stars. The relation between mass and local luminosity is then identical for the two types of object, as given in Table I.

Also listed in Table I are other properties which depend upon this assumption. Note that the entropy of interstellar gas, $S \sim 10^{10} \mathrm{erg} K^{-1} \mathrm{~g}^{-1}$, is contained within the entropy limits of such disks, while supermassive stars require much higher entropies for support. Note that the relations for the central temperature $\left(T_{9}\right)_{c}$ and effective surface temperature $T_{e}$ are similar for the two kinds of objects, except for the weak dependence on the surface density $\sigma$ of the disks. Since disks can become more relativistic than stars, this means that larger disk masses can reach a given central temperature.

Some consequences of this fact are illustrated in Figure 3, showing various lifetimes of interest. Matching the proton lifetime in the CNO cycle, $\tau_{p}(\mathrm{CNO})$, to the nuclear

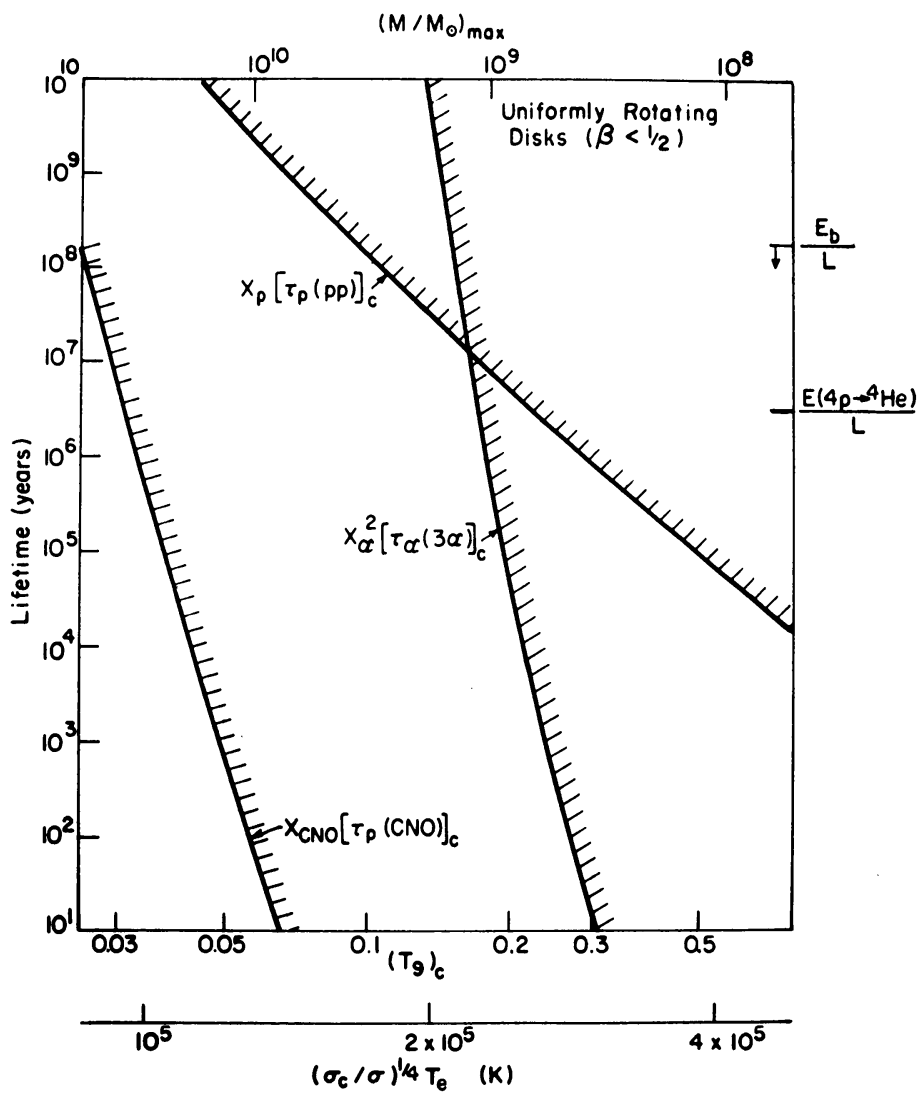

Fig. 3. Various lifetimes of importance for supermassive disks. The nuclear lifetimes due to the $p p$ chain, CNO cycle, and triple- $\alpha$ reaction are evaluated at the center of the disk. Also indicated are the effective surface temperature and maximum mass corresponding to each value of the central temperature $\left(T_{9}\right)_{c}$. 
lifetime $E\left(4 p \rightarrow{ }^{4} \mathrm{He}\right) / L$, with a normal CNO abundance, yields a maximum hydrogenburning mass of $\sim 2 \times 10^{10} M_{\odot}$, as compared with $3 \times 10^{6} M_{\odot}$ for a uniformly rotating supermassive star. It is also seen that the Kelvin-Helmholtz flattening time $E_{b} / L$ can be greater than the nuclear burning lifetime of $3 \times 10^{6} \mathrm{yr}$. The disk will flatten at constant central temperature until the gas pressure dominates, at which point it begins to cool.

Finally we apply this model to the QSO 3C 345, where it is assumed that the light variations correspond to a local rotation period of 200 days, although of course the

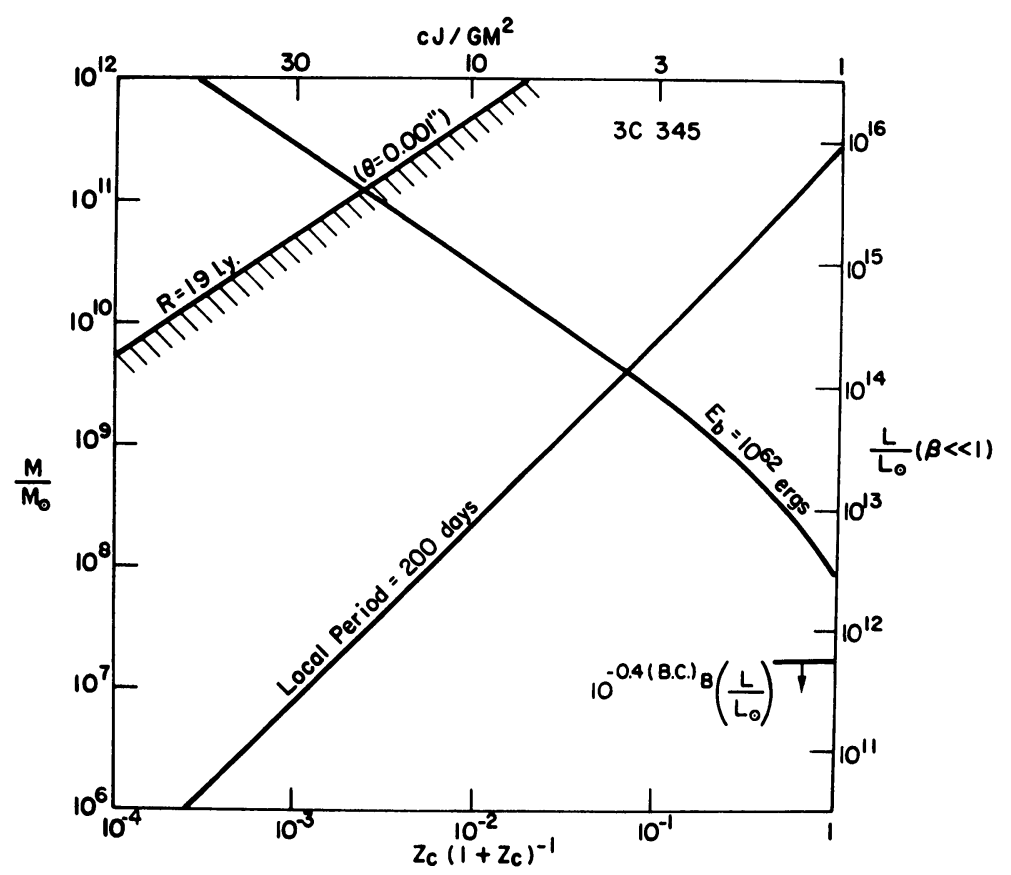

Fig. 4. Curves of constant period, binding energy, and radius of uniformly rotating disks. The values (or upper limits) chosen are related to observations of the variable QSO 3C 345. The upper limit on the amount of thermal radiation seen through the $\mathrm{B}$ filter is also indicated.

evidence is not compelling at present (Kinman et al., 1968; Morrison, 1969). In Figure 4 is shown the locus of disks having this period on the mass parameter, relativity parameter plane. Also shown is a curve of constant binding energy, taken to be near the upper limit of that thought necessary. It is seen that the limit on the radius, set by the radio interferometric result of Kellermann et al. (1968), easily allows the solution $M \sim 4 \times 10^{9} M_{\odot}$ and $z_{c} \sim 0.1$ obtained from the assumed period and estimated binding energy. (In fact, the resulting radius is 0.02 light years.) As has been pointed out by Fahlman (1970), no such solution exists for uniformly rotating supermassive stars. However, such a solution requires rather high luminosity, $L \sim 10^{14} L_{\odot}$. This can be compatible with the observational upper limit shown if either: (a) the peak 
of the spectrum is well away from the visible region, so that the bolometric correction to the observed $B$ magnitude $(B C)_{B}$ is large; or (b) the gas pressure dominates, so that the luminosity is no longer so high.

\section{References}

Bardeen, J. M. and Wagoner, R. V.: 1969, Astrophys. J. Letters 158, L65.

Cavaliere, A., Pacini, F., and Setti, G.: 1969, Astrophys. Letters 4, 103.

Cavaliere, A., Morrison, P., and Pacini, F.: 1970, Astrophys. J. Letters 162, L133.

Fahlman, G. G.: 1970, Astrophys. J. Letters 160, L87.

Fowler, W. A.: 1966, Astrophys. J. 144, 180.

Hoyle, F. and Fowler, W. A.: 1963a, Monthly Notices Roy. Astron. Soc. 125, 169.

Hoyle, F. and Fowler, W. A.: 1963b, Nature 197, 533.

Kellermann, K. I., Clark, B. G., Bare, C. C., Rydbeck, O., Ellder, J., Hansson, B., Kollberg, E., Hoglund, B., Cohen, M. H., and Jauncey, D. L.: 1968, Astrophys. J. Letters 153, L209.

Kinman, T. D., Lamla, E., Ciurla, T., Harlan, E., and Wirtanen, C. A.: 1968, Astrophys. J. $152,357$. Morrison, P.: 1969, Astrophys. J. Letters 157, L73.

Salpeter, E. E. and Wagoner, R. V.: 1971, Astrophys. J. 164, 557.

Wagoner, R. V.: 1969, Ann. Rev. Astron. Astrophys. 7, 553.

\section{Discussion}

Pachner: I am not sure whether the simplifying assumption of a uniform rotation does not substantially disturb the physical situation in rotating bodies. Since the paper of Raychaudhuri published fifteen years ago it is well-known that any deviation from an isotropic rotation increases the possibility of a gravitational collapse, even in the bodies without the uniform distribution of matter.

Wagoner: I am not familiar with all the details of the paper of Raychaudhuri. What is isotropic rotation? In any case, I know of no one who has investigated the stability against overall gravitational collapse of rapidly rotating, relativistic, isolated objects. 